\title{
Inclination Effects and Beaming in Black Hole X\# Ray Binaries
}

\section{Citation}

Narayan, Ramesh, and Jeffrey E. McClintock. 2005. "Inclination Effects and Beaming in Black Hole X\#Ray Binaries." The Astrophysical Journal 623 (2): 1017-25. https:// doi.org/10.1086/428709.

\section{Permanent link}

http://nrs.harvard.edu/urn-3:HUL.InstRepos:41384967

\section{Terms of Use}

This article was downloaded from Harvard University's DASH repository, and is made available under the terms and conditions applicable to Other Posted Material, as set forth at http:// nrs.harvard.edu/urn-3:HUL.InstRepos:dash.current.terms-of-use\#LAA

\section{Share Your Story}

The Harvard community has made this article openly available.

Please share how this access benefits you. Submit a story.

Accessibility 


\title{
INCLINATION EFFECTS AND BEAMING IN BLACK HOLE X-RAY BINARIES
}

\author{
Ramesh Narayan and JefFrey E. McClintock \\ Harvard-Smithsonian Center for Astrophysics, Cambridge, MA 02138; rnarayan@cfa.harvard.edu, jem@cfa.harvard.edu \\ Received 2004 August 4; accepted 2005 January 1
}

\begin{abstract}
We investigate the dependence of observational properties of black hole X-ray binaries on the inclination angle $i$ of their orbits. We find the following: (1) Transient black hole binaries show no trend in their quiescent X-ray luminosities as a function of $i$, suggesting that the radiation is not significantly beamed. This is consistent with emission from an accretion disk. If the X-rays are from a jet, then the Lorentz factor $\gamma$ of the jet is $<1.24$ at the $90 \%$ confidence level. (2) The X-ray binary $4 \mathrm{U} 1543-47$ with $i \sim 21^{\circ}$ has a surprisingly strong fluorescent iron line in the high soft state. Quantifying an earlier argument by Park et al., we conclude that if the continuum X-ray emission in this source is from a jet, then $\gamma<1.04$. (3) None of the known binaries has $\cos i<0.25$ or $i>75^{\circ}$. This fact, plus the lack of eclipses among the 20 black hole binaries in our sample, strongly suggests at the $99.5 \%$ confidence level that systems with large inclination angles are hidden from view. The obscuration could be the result of disk flaring, as suggested by Milgrom for neutron star X-ray binaries. (4) Transient black hole binaries with $i \sim 70^{\circ}-75^{\circ}$ have significantly more complex X-ray light curves than systems with $i \lesssim 65^{\circ}$. This may be the result of variable obscuration and/or variable height above the disk of the radiating gas.
\end{abstract}

Subject headings: accretion, accretion disks — black hole physics — radiation mechanisms: nonthermal — $\mathrm{X}$-rays: binaries

\section{INTRODUCTION}

An accretion disk is intrinsically nonspherical since the angular momentum vector of the orbiting gas specifies a unique direction. Depending on the direction from which one views the disk - face-on, edge-on, or something in between - one expects differences in what one observes. Apart from the obvious $\cos i$ factor, which determines the projected area of the disk, $i$ being the angle between the disk normal and the direction to the observer, there could be beaming effects if the radiating gas has relativistic motion. The orbital motion of the gas in the disk leads to blue- and redshifts for observers who are close to edgeon $\left(i \rightarrow 90^{\circ}\right)$, while the radiation from a jet is relativistically boosted for observers who are more face-on $\left(i \rightarrow 0^{\circ}\right)$. There may also be effects due to obscuration. For instance, the radiation emitted by one part of the disk may be blocked from the observer's view by other parts of the disk; alternatively, the secondary star may do the obscuring, in which case one expects eclipses in the light curve.

In order to study these inclination effects, we need a sample of disk systems for which we have independent estimates of $i$. $\mathrm{X}$-ray binaries are particularly useful in this regard. Because the gas accreting onto the compact star is supplied by the secondary, the angular momentum of the material is aligned with the orbital angular momentum of the binary system. Thus, the accretion disk is expected to be coplanar with the binary orbit. The inclination of the binary orbit with respect to the observer's line of sight can be estimated from the light curve of the secondary (e.g., Orosz \& Bailyn 1997). This inclination directly gives the inclination angle $i$ of the disk.

We focus in this paper on black hole X-ray binaries, limiting our study to the 20 black hole systems with dynamical mass information (McClintock \& Remillard 2005; Orosz et al. 2004; Casares et al. 2004). Of these 20 sources, 17 are transient black hole X-ray novae (BHXNs) and the remaining three are persistent sources. We collect from the literature estimates of the inclination angles of these binaries and investigate their luminosities, spectra, and light curves. Our aim is to identify any patterns or systematic variations with respect to inclination in the data. In $\S 2$ we discuss relativistic beaming effects and look for evidence of jets in certain spectral states of black hole binaries. Then, in $\S \S 3$ and 4 we discuss obscuration effects. We conclude in $\S 5$ with a summary.

\section{JETS AND RELATIVISTIC BEAMING}

Consider an accreting black hole with twin relativistic jets emitted in opposite directions. Let us make the reasonable assumption that the jets are perpendicular to the accretion disk so that the inclination angle $i$ defined earlier is also the angle between the line of sight and the jet direction. If the bulk velocity of the jets is $\beta=v / c$ and the corresponding Lorentz factor is $\gamma$, then the Doppler factors of the approaching and receding jets are given by

$$
\delta_{a}=\frac{1}{\gamma(1-\beta \cos i)}, \quad \delta_{r}=\frac{1}{\gamma(1+\beta \cos i)} .
$$

Let the spectrum of the source have the form $S_{\nu} \propto \nu^{-\alpha}$. Then the total flux density from both jets as seen by an observer at angle $i$ to the jet axis is given by (see Mirabel \& Rodríguez 1999)

$$
S_{\mathrm{obs}}(i)=S_{0}\left(\delta_{a}^{k+\alpha}+\delta_{r}^{k+\alpha}\right),
$$

where $S_{0}$ is the emitted flux density of each jet and the index $k$ takes either the value 2 (for a continuous jet) or 3 (for discrete blobs). We assume $k=2$ in what follows, both because it is more appropriate for our problem and because it gives more conservative results.

The curves in Figure 1 show the variation of $S_{\text {obs }}$ with $i$ for different choices of $\gamma$. The calculations assume $\alpha=1$ (photon index 2, as appropriate for quiescent BHXNs), and the curves have been normalized so as to have the same flux density at $\cos i=0.5$. The effect of relativistic beaming is obvious. 

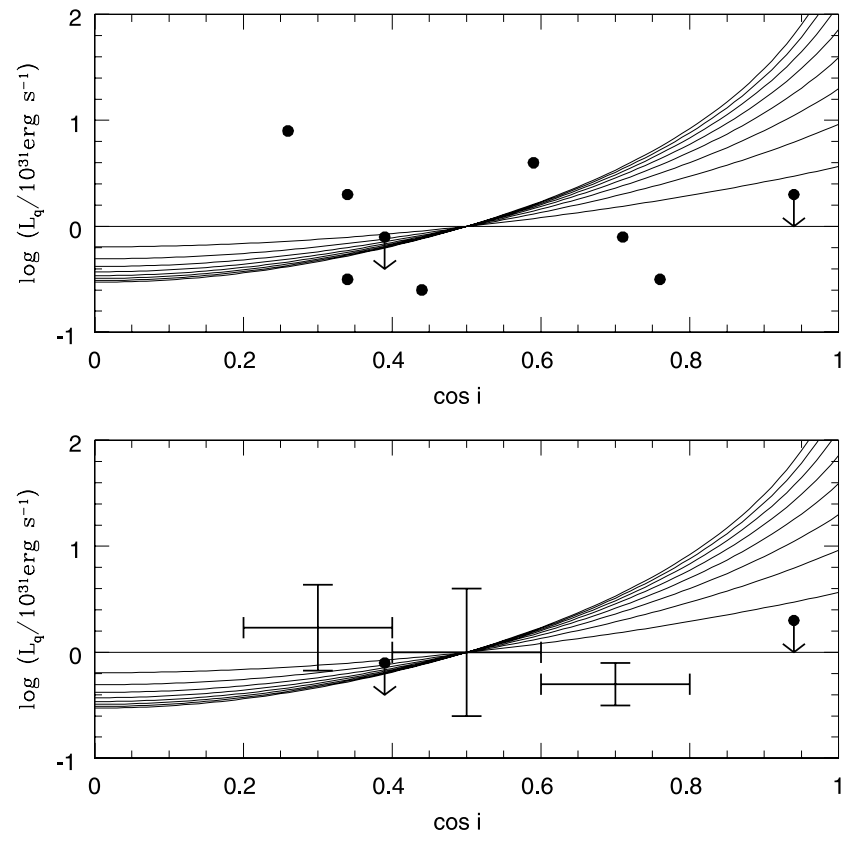

FIG. 1.-Top: Quiescent X-ray luminosities of BHXNs in units of $10^{31} \mathrm{ergs}$ $\mathrm{s}^{-1}$ plotted against the cosine of the inclination angle of the binary. The curves indicate the expected variation according to a jet model for different choices of the jet Lorentz factor: $\gamma=1.0$ (horizontal line) $1.2,1.4, \ldots, 2.6$. Bottom: The same data grouped into bins of width 0.2 in $\cos i$. Upper limits are shown separately. Both panels suggest that there is no significant relativistic beaming of the X-ray emission in quiescent BHXNs.

Especially for $\gamma>2$, the observed flux in the limit $\cos i \rightarrow 1$ (or $i \rightarrow 0$ ) is nearly 2 orders of magnitude larger than the flux in the limit $\cos i \rightarrow 0(i \rightarrow \pi / 2)$. Given a sample of sources with estimates of $i$, it might be possible to test for such a strong effect.

Below we test for the presence of jets and beaming in two different spectral states of black hole binaries. In $\S 2.1$ we describe current ideas on the nature of the low hard state and the quiescent state of BHXNs. In $\S 2.2$ we consider the luminosities of BHXNs in the quiescent state and show that there is very little evidence for jet-induced beaming in this state, consistent with earlier indications that there is no significant beaming in the low hard state. We then consider in $\S 2.3$ a particular source, $4 \mathrm{U} 1543-47$, in the high soft state and again show that we can rule out any significant beaming.

\subsection{Low Hard State and Quiescent State}

Among the five common spectral states that black hole X-ray binaries display (McClintock \& Remillard 2005), the low hard state and the quiescent state are of particular interest. These two states are believed to be similar to each other in their underlying physics, except that the quiescent state has a much lower luminosity and, by inference, a lower mass accretion rate. Both states are characterized by the presence of a power-law spectral component in hard X-rays and a near-absence of a blackbodylike component in soft X-rays (McClintock \& Remillard 2005). The latter component is expected to be dominant if the source has a traditional optically thick disk; therefore, such a disk is either absent or is energetically negligible.

In an important early paper, Shapiro et al. (1976) described a hot optically thin two-temperature accretion solution and suggested that the low hard state of Cyg X-1 may correspond to this solution. However, Pringle (1976) showed that the solution is thermally unstable. Narayan \& Yi (1995) and Abramowicz et al. (1995) demonstrated that there are, in fact, two distinct hot solutions: (1) the solution discovered by Shapiro et al. (1976), and (2) a second two-temperature solution that is characterized by energy advection. The latter solution, which was originally discovered by Ichimaru (1977), is thermally stable. It is named an advection-dominated accretion flow (ADAF) and has been used for a number of years to model the low and quiescent states of black hole binaries (Narayan et al. 1996; Esin et al. 1997, 1998, 2001; McClintock et al. 2003; see Narayan et al. 1998 for a review). According to this model, a black hole binary in the low or quiescent state has a standard optically thick disk only at relatively large radii, while the gas at smaller radii is in the form of an ADAF. The X-ray emission is produced by the ADAF via Comptonization of soft photons.

There are two sources of soft photons in the above model: synchrotron emission from the hot electrons in the ADAF, and thermal photons from the thin accretion disk on the outside (see Narayan et al. 1997; Esin et al. 1997). Depending on the location of the transition radius between the ADAF and the outer disk, one or the other dominates the Comptonization. Generally, synchrotron emission dominates in the quiescent state and thermal radiation from the outer disk dominates in the low hard state (and even more so in the intermediate state between the low hard state and the high soft state).

While the ADAF model provides a satisfactory description of the X-ray emission in black hole X-ray binaries, it is unable to explain the radio emission that is often observed in the low hard state. This is not surprising since the radio emission has been resolved into a jet in Cyg X-1 and jets are inferred for other sources via a brightness temperature argument (Fender 2005). Markoff et al. (2001) proposed a model for XTE J1118+480 in which nearly the entire spectrum from radio to hard X-rays is produced by synchrotron emission from a jet. Corbel et al. (2003) noted that there is a strong correlation between the radio and X-ray emission in the black hole binary GX 339-4 in the low hard state and the quiescent state and suggested that a significant fraction of the observed X-ray radiation in these two states is produced by synchrotron emission from a jet. In this picture, the hot ADAF is virtually silent in all bands.

Several studies have followed up on this suggestion. Heinz \& Sunyaev (2003) worked out a scaling relation between the synchrotron flux at a given frequency, the mass of the black hole, and the mass accretion rate. Their model is applicable to jets anchored in either an ADAF or a standard disk. Merloni et al. (2003) extended this work and showed that accreting black holes follow quite well a "fundamental plane" in the threedimensional parameter space of radio luminosity, X-ray luminosity, and black hole mass. However, they came down in favor of the ADAF rather than the jet as the source of the X-ray emission in the low hard state. Falcke et al. (2004) argued instead that synchrotron emission from the jet is the source of the X-rays. In a recent paper, Heinz (2004) has presented additional arguments why a synchrotron jet is unlikely to explain the X-ray emission in low hard state binaries.

In the context of spectral modeling, a jet has two important features. First, the radiating particles are expected to be highly nonthermal; this is usually modeled with a power-law distribution in energy. A nonthermal distribution is somewhat problematic for explaining the X-ray emission since the hard X-ray spectra of several low hard state binaries (e.g., Esin et al. 1998) show a turnover at about $100 \mathrm{keV}$. This turnover is easily explained in the ADAF model, since the thermal electrons naturally acquire temperatures of this order (Esin et al. 1997, 1998; Zdziarsky et al. 2003). For a wide range of accretion rates, the ADAF model gives electron temperatures that vary by only a 
TABLE 1

InCLINATIONS AND QUiescent Luminosities of BHXNs

\begin{tabular}{|c|c|c|c|c|c|}
\hline $\begin{array}{l}\text { System } \\
\text { (1) }\end{array}$ & $\begin{array}{c}P_{\text {orb }} \\
(\mathrm{hr}) \\
(2)\end{array}$ & $\begin{array}{c}i \\
\text { (deg) } \\
(3)\end{array}$ & $\begin{array}{c}D \\
(\mathrm{kpc}) \\
(4)\end{array}$ & $\begin{array}{c}\log \left[L_{q}\left(\mathrm{erg} \mathrm{s}^{-1}\right)\right]^{\mathrm{a}} \\
(5)\end{array}$ & $\begin{array}{c}\text { References } \\
\text { (6) }\end{array}$ \\
\hline 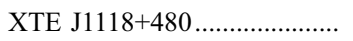 & 4.1 & $70_{-7}^{+3}$ & 1.8 & 30.5 & 1,2 \\
\hline GRO J0422+32 …...................... & 5.1 & $45 \pm 2$ & 2.6 & 30.9 & 3,4 \\
\hline 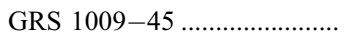 & 6.8 & $67(?)$ & 5.0 & $<30.9^{\mathrm{b}}$ & 5,6 \\
\hline 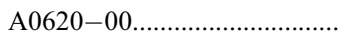 & 7.8 & $40.8 \pm 3$ & 1.0 & 30.5 & 4,7 \\
\hline GS2000+25 & 8.3 & $64 \pm 1.3$ & 2.7 & 30.4 & 4,5 \\
\hline GS/GRS 1124-683 .................... & 10.4 & $54_{-15}^{+4}$ & 5.5 & 31.6 & 8,9 \\
\hline 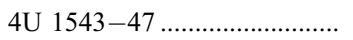 & 26.8 & $20.7 \pm 1.0$ & $7.5^{\mathrm{c}}$ & $<31.3^{\mathrm{b}}$ & 4,10 \\
\hline GRO J1655-40 .......................... & 62.9 & $70.2 \pm 1.9$ & 3.2 & 31.3 & 4,11 \\
\hline SAX J1819.3-2525 .................. & 67.6 & $75 \pm 2$ & 9.6 & 31.9 & $5,12,13$ \\
\hline GS $2023+338 \ldots \ldots \ldots \ldots \ldots \ldots$ & 155.3 & $56 \pm 4$ & 3.5 & 33.1 & 4,14 \\
\hline
\end{tabular}

${ }^{\text {a }}$ Quiescent luminosity in the $0.5-10 \mathrm{keV}$ band.

${ }^{\mathrm{b}}$ Upper limits are at the $95 \%$ level of confidence.

${ }^{c}$ Distance obtained from reference 10 .

ReFerenCES.- (1) J. Orosz et al. 2005, in preparation; (2) McClintock et al. 2003; (3) Gelino \& Harrison 2003; (4) Garcia et al. 2001; (5) Orosz 2002; (6) Hameury et al. 2003; (7) Gelino et al. 2001b; (8) Gelino et al. 2001a; (9) Sutaria et al. 2002; (10) J. Orosz et al. 2005, in preparation; (11) Greene et al. 2001; (12) Tomsick et al. 2003; (13) Orosz et al. 2001; (14) Shahbaz et al. 1994.

factor of a few. The reason is that thermal Comptonization becomes significantly more effective when the electrons are relativistic. There is hence a natural thermostat in these models whereby the electrons tend to equilibrate at $k T_{e}$ a factor of a few less than $m_{e} c^{2}$. Correspondingly, the Compton emission cuts off at $\sim 100-200 \mathrm{keV}$. In the jet synchrotron model, there is nothing special about a photon energy of $100 \mathrm{keV}$, and so the observed turnover of the spectra at this energy requires some degree of fine-tuning.

The second feature of a jet - one that is unique to it - is that it involves relativistic bulk motion. The jets seen in blazars, radio galaxies, and quasars often have bulk Lorentz factors $\gamma \sim 10$, resulting in strong beaming of the radiation. The radio jets seen in some X-ray binaries in the very high state, the microquasars, have $\gamma \sim 2.6$ ( Mirabel \& Rodríguez 1999), which again implies considerable beaming. If the X-ray emission in black hole binaries in the quiescent and low hard states is from a jet, one might naively expect the radiation to be strongly beamed. However, recent X-ray and radio studies of BHXNs argue against such strong beaming in the low hard state (Gallo et al. 2003; Maccarone 2003). In $\S 2.2$ we consider the quiescent state of these sources and investigate what limits we can place on beaming.

\subsection{Black Hole X-Ray Novae in Quiescence}

Table 1 lists the parameters of 10 well-studied BHXNs in order of increasing orbital period. The inclination angles $i$ listed in column (3) are taken for each source from the first reference cited. The following reference or references support the distance and luminosity data given in columns (4) and (5). The distances, apart from GRO J1655-40 (Hjellming \& Rupen 1995), are based on a dynamical model for each binary system that allows a determination of the physical radius of the secondary star, and hence its absolute visual magnitude, given its spectral type (e.g., Barret et al. 1996). Taking into account a correction for interstellar reddening and a correction for the continuum flux that is contributed by the accretion disk, one obtains the apparent dereddened magnitude of the secondary, and hence the distance. The uncertainty in the distances so obtained is typically $\pm 25 \%$ (Barret et al. 1996). We note that these distances have been criticized as underestimates by Jonker
\& Nelemans (2004) on what we consider speculative grounds; moreover, since those authors do not recommend a set of corrected distances, we have no choice but to use the standard distances listed in Table 1. The luminosity column gives for each source the lowest (absorption-corrected) X-ray luminosity that has been reported in observations made using either Chandra or XMM-Newton.

The values of inclination are of central importance to this work. In our selection of the data, we have favored values determined by modeling IR light curves because they are less contaminated than optical light curves by emission from the accretion disk. However, even the inclinations derived from IR light curves are subject to criticism, as shown for example by the debate over the proper interpretation of the light curves of A0620-00 (Shahbaz et al. 1999; Froning \& Robinson 2001; Gelino et al. 2001b). Nevertheless, the spectral energy distributions (BVRIJHK) obtained for some of the sources provide a limit on the contamination and a convincing lower limit on the inclination angle (e.g., Gelino et al. 2001b; Gelino \& Harrison 2003). Moreover, in a few favorable cases such as GRO J1655-40, the nonstellar flux is a small fraction of the stellar flux (Greene et al. 2001). Finally, the agreement between the inclinations given in Table 1 and most of the earlier and less precise determinations gives one reasonable confidence in the values we have adopted (e.g., in the case of GS/GRS 1124-68 [see Orosz et al. 1996; Shahbaz et al. 1997]).

The top panel in Figure 1 shows the quiescent X-ray luminosities of the BHXNs from Table 1 plotted against the inclinations $i$ of their binary orbits. The luminosities have been normalized by $10^{31} \mathrm{ergs} \mathrm{s}^{-1}$ for easy comparison with the normalized theoretical curves. The source GS 2023+338 (or V404 Cyg) has not been shown in the plot. This source has a subgiant companion and is much brighter than the other BHXNs; in fact, it is off-scale on the plot. Fortunately, the source has a value of $\cos i \sim 0.5$ (the normalization point or pivot point of the theoretical curves), so it does not bias the results.

We see from Figure 1 that there is no hint in the data for any increase in the observed luminosity of low-inclination systems ( $\cos i \rightarrow 1$ ). Indeed, the most pole-on system in the sample, $4 \mathrm{U} 1543-47$ with $i=20.7 \pm 1.0$, has a $95 \%$ confidence upper limit on its quiescent luminosity that is below the luminosity 
predicted for all values of $\gamma$ (other than unity) considered in the plot. The bottom panel in Figure 1 shows the same data binned in $\cos i$. In each bin, all the systems with measured luminosities in that bin have been combined and their mean luminosity has been plotted, along with the estimated error in the mean. The systems with upper limits are shown separately. This plot confirms that there is no evidence for any increase in the observed luminosity with increasing cos $i$. If quiescent $\mathrm{BHXN}$ s produce their X-ray emission from a jet, then it would appear that the Lorentz factor of the jet must be fairly small.

Note the special importance of 4U 1543-47. With the lowest inclination among all our sources, it has the greatest ability to test for the presence of a jet. The source has a relatively low luminosity, but is this significant? The answer is not obvious since Figure 1 indicates that the quiescent luminosities of BHXNs in general have a large dispersion. We attempt to provide a quantitative analysis of this question here.

Leaving aside GS 2023+338 (see above), the seven objects in Figure 1 with measured quiescent luminosities have a mean X-ray luminosity of $\log L_{q}=31$ and a dispersion around the mean of $\sigma_{\log L}=0.6$. A fraction of the dispersion may be caused by beaming, but most of the dispersion seems to be intrinsic to the sources (see also Heinz \& Merloni 2004 for a related discussion). For simplicity, and also to be maximally conservative, we assume that the entire dispersion is intrinsic to the sources. Now, given a value for the jet Lorentz factor $\gamma$, and using the estimated inclination angle of $i=20^{\circ} .7$ in $4 \mathrm{U} 1543-47$, we can estimate the mean expected luminosity of the source $\left\langle\log L_{q}(\gamma)\right\rangle$. (The theoretical curves in Fig. 1 show this quantity for selected values of $\gamma$.) Assuming a lognormal probability distribution for $\log L_{q}$ with dispersion $\sigma_{\log L}=0.6$, we then obtain the following probability distribution for $\log L_{q}$ of $4 \mathrm{U} 1543-47$ :

$$
\begin{aligned}
P\left(\log L_{q} ; \gamma\right) & =\frac{1}{\sigma_{\log L} \sqrt{2 \pi}} \\
& \times \exp \left\{-\frac{\left[\log L_{q}-\left\langle\log L_{q}(\gamma)\right\rangle\right]^{2}}{2 \sigma_{\log L}^{2}}\right\} d \log L_{q} .
\end{aligned}
$$

This distribution is a function of $\gamma$ because the quantity $\left\langle\log L_{q}(\gamma)\right\rangle$ depends on it.

Garcia et al. (2001) observed 4U 1543-47 for 9.9 ks with Chandra. From the estimated distance to the source, they state that a luminosity of $L_{q}=3.0 \times 10^{31} \mathrm{ergs} \mathrm{s}^{-1}$ corresponds to 5 counts; i.e., a luminosity of $L_{q}=6.0 \times 10^{30} \mathrm{ergs} \mathrm{s}^{-1}$ is equivalent to 1 count. In the actual observations, Garcia et al. detected no counts at all. Assuming Poisson statistics, the probability of detecting zero counts for a source luminosity $L_{q}$ is equal to $\exp \left(-L_{q} / 6.0 \times 10^{30}\right)$. Combining this with the probability distribution for $\log L_{q}$ written above, we obtain the probability of measuring zero counts for a given jet $\gamma$ to be

$$
\begin{aligned}
P(0 \text { counts } ; \gamma)=\frac{1}{\sigma_{\log L} \sqrt{2 \pi}} \int \exp \{ & -\frac{\left[\log L_{q}-\left\langle\log L_{q}(\gamma)\right\rangle\right]^{2}}{2 \sigma_{\log L}^{2}} \\
& \left.-\frac{L_{q}}{6.0 \times 10^{30}}\right\} d \log L_{q} .
\end{aligned}
$$

Doing the integral numerically, we find that the probability is 0.099 for $\gamma=1.24$; i.e., we can reject this value of $\gamma$ (or anything larger) at the $90 \%$ confidence level. Similarly, we can reject $\gamma \geq$ 1.41 at the $95 \%$ confidence level. These results help to quantify the qualitative impression one obtains from Figure 1 that the quiescent X-ray luminosity of 4U 1543-47 is inconsistent with significant jet beaming. Note that the upper limit on the quiescent luminosity of $4 \mathrm{U} 1543-47$ was obtained with a relatively short observation. More sensitive X-ray observations could provide a much more stringent limit.

Gallo et al. (2003) obtained an upper limit of $\gamma \sim 2$ for the radio-emitting gas in the low hard state, while Maccarone (2003) deduced $\gamma \lesssim 1.4$ for the X-ray-emitting gas in GRO J1655-40. To the extent that the quiescent state is similar to the low state, the limit $\gamma \leq 1.24$ in the present study confirms these earlier indications. Equally, however, the evidence supports a jet-ADAF model for low-state and quiescent-state BHXNs. In this model, the radio and other long-wavelength emission is from a jet, but the high-energy radiation, especially the hard X-rays, is from an ADAF. Malzac et al. (2004) and Yuan et al. (2004) have shown that such a model is consistent with the spectral and timing data on XTE J1118+480 in the low state.

In jet models of the X-ray emission, it is proposed that both the radio and X-ray emission are from the jet, although not from the same region. While the radio is emitted farther out by fastmoving gas, the X-rays are postulated to come from the "base of the jet," where the gas is moving more slowly. It is not clear to the present authors that the slowly moving X-ray-emitting gas in these models is really distinct from the hot gas in the underlying ADAF. If the arguments against a synchrotron origin of the X-ray emission from the jet described in $\S 2.1$ are correct, then the jet model will need to invoke Compton scattering to produce the X-rays. The model would then be so similar to the jet-ADAF model that it may be different only in name.

While we have focused primarily on emission from a jet in the results presented in Figure 1, it should be noted that even a pure accretion model does involve moderately relativistic flow near the black hole. However, the velocities are unlikely to exceed about $c / 2$, and hence $\gamma$ is at most $\sim 1.1-1.2$. The predicted effect due to beaming is then too small to be distinguished with our present small sample of sources. Because of the different geometry, the beaming due to an optically thin axisymmetric accretion flow such as an ADAF will give a larger luminosity as $\cos i \rightarrow 0$ compared to $\cos i \rightarrow 1$, exactly opposite to the effect with a jet. With a large enough sample one might be able to identify this dependence.

Finally, we note that the inclination angles listed in Table 1 refer to the binary orbit, which we have argued is likely to be coplanar with the large-scale accretion disk. However, none of the measurements directly give the orientation of the jet. In order to obtain the latter, we made the additional assumption that the jet is oriented perpendicular to the disk. How reasonable is this?

Independent information on the jet orientation is available for two sources, GRO J1655-40 and SAX J1819.3-2525. Hjellming \& Rupen (1995) estimated the jet angle in GRO $\mathrm{J} 1655-40$ to be $85^{\circ}$, while the binary inclination angle is measured to be $70^{\circ}$ (Table 1). The jet is thus misaligned from the disk normal by $\sim 15^{\circ}$, which is a relatively small angle and has little effect on our conclusions. However, SAX J1819.3-2525 is more problematic. In this source, Orosz et al. (2001) interpreted the radio observations of Hjellming et al. (2000) to deduce a jet angle $<10^{\circ}$, compared to a binary inclination of $75^{\circ}$. The misalignment between the jet and the normal to the disk is thus more than $60^{\circ}$, which is enormous. Maccarone (2002) has shown that the timescale on which the spin axis of the black hole (which is presumably the same as the direction of the jet) and the angular momentum axis of the disk become aligned can be comparable to or longer than the lifetime of the binary for 
transient sources with a low-outburst duty cycle. Therefore, such a large misalignment could survive if it was somehow created initially when the black hole was formed.

If the jet orientation in SAX J1819.3-2525 is indeed offset by a large angle from the disk normal, then the conclusions reached in this section regarding the presence/absence of jets and relativistic beaming in the quiescent state of BHXNs become less compelling. (The conclusions reached in $\S \S 3$ or 4 are not affected.) We note, however, that there are alternate explanations of the observations in SAX J1819.3-2525 that do not require a small jet angle. The reason is that the radio observations of this source did not directly measure the proper motion of the jet. All that was measured was the angular offset between the peak of the jet radio emission and the position of the binary. If the jet was ejected during the X-ray outburst that occurred a short time prior to the radio observations, then the position offset implies the jet angle quoted above: $<10^{\circ}$. However, as Chaty et al. (2001) have argued, the jet might have been ejected a couple of weeks earlier than the X-ray outburst, in which case the jet would have had a modest Lorentz factor and its orientation would be consistent with the measured binary inclination. This possibility becomes all the more reasonable considering that the source showed optical activity for more than a month prior to the X-ray outburst.

\section{3. $4 U$ 1543-47 in the High Soft State}

Park et al. (2004) pointed out that, during the 2002 accretion outburst of $4 \mathrm{U} 1543-47$, the Fe line from the disk in this source had an average equivalent width of $\sim 300 \mathrm{eV}$, which is 3 times larger than the predicted equivalent width (George \& Fabian 1991) for an unbeamed source. Since it is unlikely that the theoretical prediction is off by more than a factor of a few, Park et al. argued that the amount of beaming allowed in the source is strongly constrained. Figure 2 quantifies this argument.

We assume that the hard X-rays irradiating the disk are produced in a jet. We then calculate the ratio of the X-ray flux density $S_{\text {obs }}$ seen by the observer at $i=21^{\circ}$ (the orbital inclination of $4 \mathrm{U} 1543-47$ ) to the mean flux density $S_{\text {irr }}$ over the range of angles $i=135^{\circ}-180^{\circ}$; the latter quantity is a measure of the flux irradiating the disk, assuming that the irradiation occurs over a cone of half-angle $45^{\circ}$ around the backward direction. We take the energy spectral index to be $\alpha=1.5$ in accordance with the observations of Park et al. (2004). We make the further reasonable assumption that the George \& Fabian (1991) estimate of the Fe line equivalent width is correct to within an order of magnitude; i.e., the equivalent width in the absence of beaming is no larger than $1000 \mathrm{eV}$. Relativistic beaming will cause the equivalent width to decrease for an observer at low inclination. This is because the observed hard $\mathrm{X}$-ray continuum will increase as a result of relativistic beaming, whereas the flux irradiating the disk (and producing the fluorescent line) will decrease. Since the observed equivalent width is about $300 \mathrm{eV}$, the maximum value allowed for the quantity $S_{\text {obs }} / S_{\text {irr }}$ in Figure 2 is $(1000 \mathrm{eV}) /(300 \mathrm{eV})=10 / 3$. This level is shown by the dotted line.

We see from Figure 2 that any X-ray-emitting jet in $4 \mathrm{U} 1543-47$ in the high soft state must have $\gamma<1.04$. This is a rather tight limit. However, the result refers to a single source and should perhaps not be taken as a general indication for all sources in the high soft state. Also, note that we are discussing here the high soft state, which is very different from the low and quiescent states discussed earlier. The model of Cyg X-1 proposed by Beloborodov (1999) and Malzac et al. (2001), in which the hard X-rays are produced in an outflowing

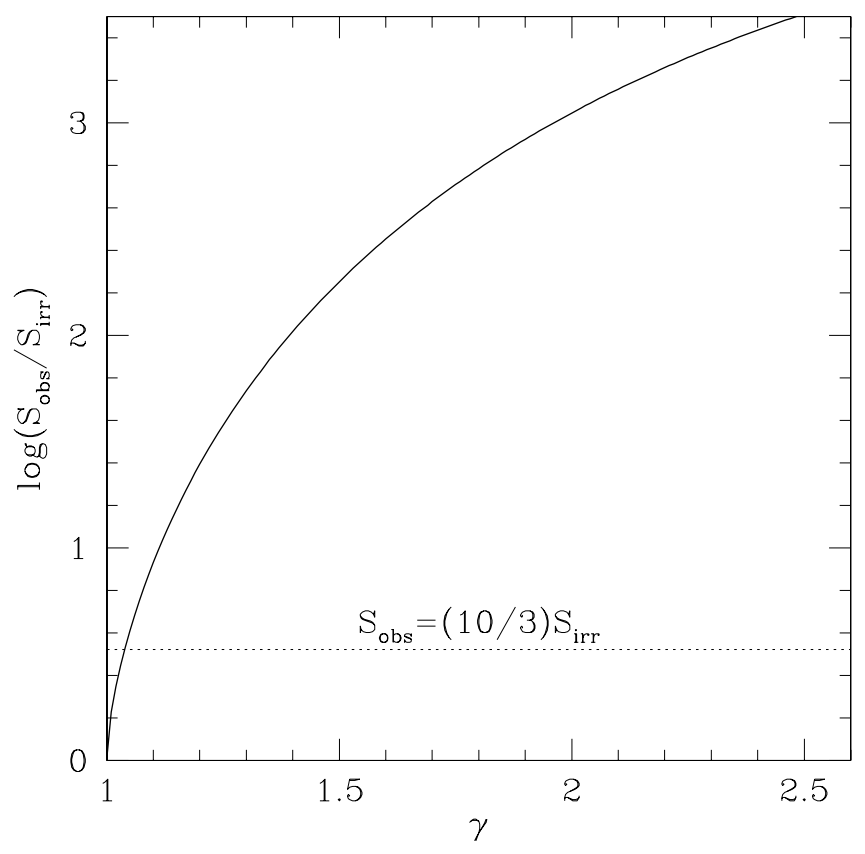

FIG. 2.-Ratio of X-ray flux density from a jet in 4 U $1543-47$ toward the observer to the mean flux density toward the accretion disk, plotted as a function of the jet Lorentz factor $\gamma$. The horizontal dotted line shows the upper limit on the ratio as estimated from the equivalent width of the Fe line. If the hard X-rays in $4 \mathrm{U} 1543-47$ during outburst are produced in a jet, then $\gamma$ must be close to unity.

corona, has a value of $\gamma$ roughly consistent with the limit derived here.

\section{ON THE ABSENCE OF EDGE-ON SYSTEMS}

An interesting feature of the sample of sources in Table 1 and Figure 1 is the absence of systems with $\cos i<0.25$, or $i>75^{\circ}$. Since $\cos i$ is proportional to the solid angle, it appears that fully $25 \%$ of the solid angle is for some reason not populated with sources. We quantify this argument here.

In addition to the 10 sources in Table 1, let us include also XTE J1550-564, which does not have a quiescent luminosity measurement and therefore is not listed in Table 1, but it does have an estimated inclination angle: $i=72^{\circ} \pm 5^{\circ}$ (Orosz et al. 2002). What is the probability that these 11 sources would by chance all have $\cos i>0.25$ ? The answer is $0.75^{11}=0.042$. That is, the absence of sources with high inclinations ( $\cos i<$ $0.25, i>75^{\circ}$ ) is significant at the $96 \%$ confidence level, a fairly strong result. The conclusion does, however, require that our estimates of inclination angles be reliable.

We now present an independent argument for the scarcity of highly inclined systems. This second argument does not require inclination estimates but is based just on the fact that no black hole X-ray binary has so far been found to eclipse. If the X-rayemitting inner accretion disks in black hole binaries are visible from all directions, then occasionally we ought to have a nearly edge-on binary for which we should see X-ray eclipses. The solid angle over which eclipses will be visible is determined by the eclipse angle $\theta_{e}$ corresponding to a ray from the X-ray source that just grazes the top (or bottom) of the secondary. For Roche lobe-filling secondaries, $\theta_{e}$ depends only on the mass ratio $Q \equiv M_{\mathrm{BH}} / M_{s}$, where $M_{\mathrm{BH}}$ is the mass of the black hole and $M_{s}$ is the mass of the secondary. Table 2 lists all 20 dynamical black hole binaries known today, along with estimates of the mass ratio $Q$. The sources are collected into four groups. 
TABLE 2

Grazing Eclipse Angles of Black Hole X-Ray Binaries

\begin{tabular}{|c|c|c|c|c|c|}
\hline $\begin{array}{l}\text { System } \\
\text { (1) }\end{array}$ & $\begin{array}{c}P_{\text {orb }} \\
(\mathrm{hr})^{\mathrm{a}, \mathrm{b}} \\
(2)\end{array}$ & $Q=M_{\mathrm{BH}} / M_{s}^{\mathrm{a}}$ & $\begin{array}{c}\theta_{e} \\
(\mathrm{deg}) \\
(4)\end{array}$ & $\begin{array}{c}\left\langle\theta_{e}\right\rangle \\
(5)\end{array}$ & $\begin{array}{c}\text { References } \\
\text { (6) }\end{array}$ \\
\hline XTE J1118+480. & 4.1 & $22.7-28.8$ & $81.5-82.1$ & 81.8 & 1 \\
\hline GRO J0422+32. & 5.1 & $3.2-13.2$ & $74.6-79.9$ & 78.3 & 1 \\
\hline GRS 1009-45. & 6.8 & $6.3-8.0$ : & $77.4-78.3$ & 77.9 & 1 \\
\hline A0620-00........ & 7.8 & $13.3-18.3$ & $80.0-80.9$ & 80.5 & 1 \\
\hline GS2000+25. & 8.3 & $18.9-28.9$ & $81.0-82.1$ & 81.6 & 1 \\
\hline GS/GRS $1124-683 \ldots \ldots \ldots \ldots . . . .$. & 10.4 & $4.8-8.8$ & $76.3-78.6$ & 77.7 & 1 \\
\hline $4 \mathrm{U} 1543-47 \ldots \ldots$ & 26.8 & $3.2-4.0$ & $74.6-75.6$ & 75.1 & 1 \\
\hline 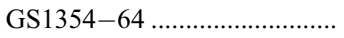 & 61.1: & $6.7-9.1$ & $77.6-78.7$ & $78.1^{\mathrm{c}}$ & 2 \\
\hline GRO J1655-40 …................... & 62.9 & $2.4-2.7$ & $73.3-73.9$ & 73.6 & 1 \\
\hline SAX J1819.3-2525 .............. & 67.6 & $2.22-2.39$ & $73.0-73.3$ & 73.1 & 1 \\
\hline GS $2023+338 \ldots \ldots \ldots \ldots \ldots \ldots \ldots \ldots \ldots \ldots \ldots \ldots \ldots \ldots$ & 155.3 & $16.1-18.9$ & $80.5-81.0$ & 80.8 & 1 \\
\hline 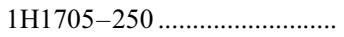 & 12.5 & $>18.9$ & $>81.0$ & $(82.2)$ & 1 \\
\hline XTE J1550-564 ...................... & 37.0 & $>12$ & $>79.6$ & $(82.2)$ & 1 \\
\hline XTE J1650-500 ..................... & 7.7 & $\ldots$ & $\ldots$ & $(77.8)$ & 3 \\
\hline XTE J1859+226...................... & 9.2: & $\ldots$ & $\ldots$ & $(77.8)$ & 1 \\
\hline 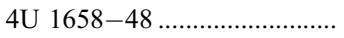 & 42.1: & $\ldots$ & $\ldots$ & $(77.8)$ & 4 \\
\hline 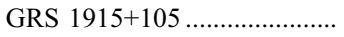 & 804.0 & $\ldots$ & $\ldots$ & $(77.8)$ & 1 \\
\hline 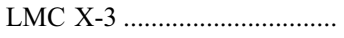 & 40.9 & $1.1-2.0$ & $69.5-72.5$ & 71.2 & 1 \\
\hline 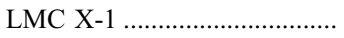 & 101.5 & $0.3-0.7$ : & $62.3-67.0$ & 65.2 & 1 \\
\hline Cyg X-1 .......... & 134.4 & $0.50-0.57$ & $66.9-67.6^{\mathrm{d}}$ & $67.2^{\mathrm{d}}$ & 1 \\
\hline
\end{tabular}

${ }^{\text {a }}$ Colon denotes uncertain value.

b For orbital period data, see McClintock \& Remillard (2005) and references 2, 3, and 4.

c Based on the best-fit value for the mass ratio, $Q=7.7$.

d The radial fraction of the Roche lobe that is filled by the star is assumed to be 0.8 (see text).

References.-(1) Orosz 2002; (2) Casares et al. 2004; (3) Orosz et al. 2004; (4) Hynes et al. 2003.

The first group in Table 2 consists of the 10 BHXNs listed in Table 1 plus the BHXN GS1354-64. These sources all have estimates of $Q$. Using the given values of $Q$, we have used the Eclipsing Light Curve code (ver. 2) described in Orosz \& Hauschildt (2000), which incorporates full Roche geometry, to obtain the ranges of $\theta_{e}$ given in column (4). Column (5) gives the mean eclipse angle $\left\langle\theta_{e}\right\rangle$, which is the value of $\theta_{e}$ corresponding to the mean $Q$ from column (3). (This is slightly more conservative than using the mean $\theta_{e}$ from col. [4].) For each binary, the probability that it will not be seen as an eclipsing source is equal to $1-\cos \left\langle\theta_{e}\right\rangle$. The mean value of this quantity for the 11 sources is 0.792 . The probability that none of the 11 would be an eclipsing source is the product of the $1-\cos \left\langle\theta_{e}\right\rangle$ factors of the individual sources, which gives 0.0766 .

The second group in Table 2 lists two BHXNs for which we have only lower limits on $Q$. If we conservatively take $Q$ to be 30 for these two sources (a larger value than for any of the sources in the first group), we obtain the estimated value $\left\langle\theta_{e}\right\rangle=82^{\circ} .2$ shown in parentheses in column (5). The third group in Table 2 consists of four BHXNs for which we have no information on $Q$. For these sources, we assume that their $Q$ values are similar to those of the first group and that their mean value of $1-\cos \left\langle\theta_{e}\right\rangle$ is therefore 0.792 . This leads to the estimated value $\left\langle\theta_{e}\right\rangle=77^{\circ} .8$ given in column (5).

Finally, the fourth group consists of three persistent sources (these are not BHXNs). In view of the high X-ray luminosity of LMC X-3 and the fact that an accretion disk is likely to be present in this source, Kuiper et al. (1988) argued that the secondary probably fills its Roche lobe. LMC X-1 is almost as luminous, so this source too probably fills its Roche lobe. We thus obtain the values of $\left\langle\theta_{e}\right\rangle$ given in Table 2 for these two sources. Cyg X-1 probably underfills its Roche lobe, but the degree to which it does so is uncertain. Bolton (1975) consid- ered a radius range for the secondary from $0.9 R_{L}-1 R_{L}$, where $R_{L}$ is the radius of the Roche lobe, and Bolton (1986) considered the range $0.8 R_{L}-1 R_{L}$. To be conservative, we have assumed $0.8 R_{L}$ in calculating the value of $\left\langle\theta_{e}\right\rangle$.

Combining all 20 sources listed in Table 2, the probability that none of them would be an eclipsing source is given by the product of the individual $1-\cos \left\langle\theta_{e}\right\rangle$ values. This gives 0.00535 . That is, the absence of eclipses is significant at the $99.5 \%$ level. This is a surprisingly strong result considering that we have assumed nothing more than that the secondaries fill their Roche lobes (except in the case of Cyg X-1; see above). Note that the probability of observing an eclipse varies like $Q^{-1 / 3}$ for large $Q$, so the $Q$ values given in Table 2 would need to be increased by a very large factor before the absence of eclipses will be consistent with a normal statistical fluctuation.

We thus have two quite independent arguments, both suggesting that the inner accretion disks in black hole X-ray binaries are not equally visible from all directions. They must be completely hidden, or at least strongly absorbed, when viewed nearly edge-on. A likely explanation is that the disks are flared by about $\pm 15^{\circ}$ and thus permanently occult the $\mathrm{X}$-rays in more nearly edge-on systems. The flare angle must be comparable to or larger than $\pi / 2-\theta_{e}$. This possibility was suggested many years ago by Milgrom (1978) to explain the rarity of eclipses in neutron star X-ray binaries. We suggest that the same explanation applies also to black hole systems. Incidentally, we use the word "flare" here in a generalized sense so as to include other geometries, such as disk "corrugation" or a "warp."

\section{DEPENDENCE OF TRANSIENT LIGHT CURVES ON INCLINATION}

If edge-on systems are obscured from sight and face-on sources are not, what about sources that are close to being obscured? 

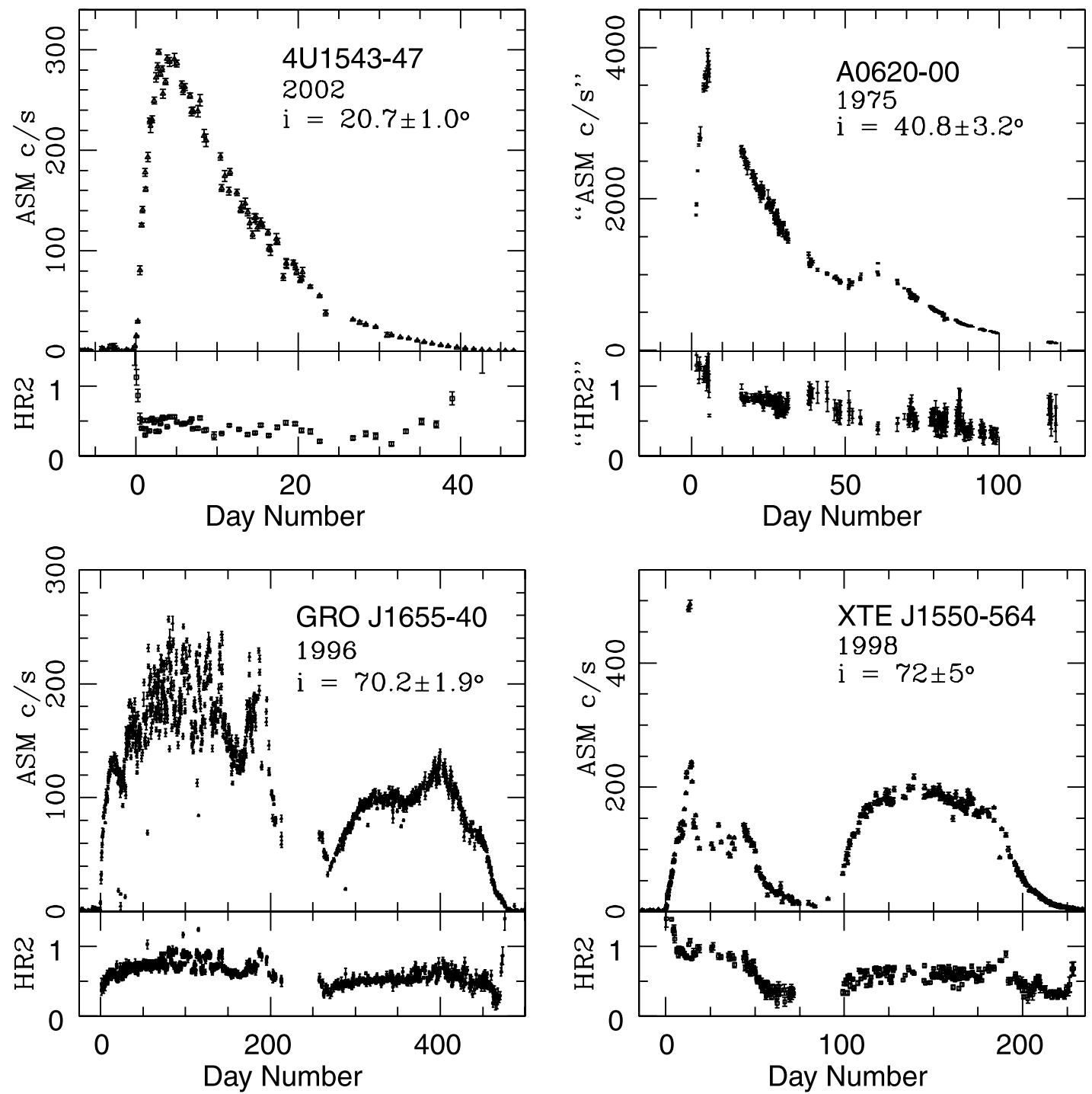

FIG. 3.- Simple X-ray light curves observed for BHXNs at low inclination (top two panels) are compared to the complex light curves observed near $i \sim 70^{\circ}$ (bottom two panels). The more recent outbursts of three of the sources (4U 1543-47, XTE J1550-564, and GRO J1655-40) were observed with the All-Sky Monitor (ASM)

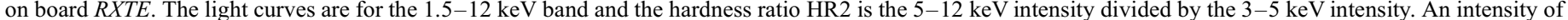
$1 \mathrm{Crab}$ corresponds to $75.5 \mathrm{ASM}$ counts $\mathrm{s}^{-1}$. Time zero is the discovery date of the outburst in question. The $1.5-15 \mathrm{keV}$ light curve of A0620-00 was obtained with the "center slat" detector on board $S A S-3$, and the ratio of the 6-15 to the $1.5-6 \mathrm{keV}$ intensities defines the $S A S-3$ hardness ratio (Buff et al. 1977). These quantities have been normalized to correspond approximately with the $R X T E$ values of ASM intensity and hardness ratio.

These would be the sources with inclination angles in the range say $70^{\circ}-75^{\circ}$ in Table 1 . Do these sources show any hint that they are close to being obscured? Here we focus on one signature: an intriguing correlation between the inclination angles of BHXNs and the complexity of their outburst light curves. In addition to the 10 sources in Table 1, we also include XTE J1550-564, with $i=72^{\circ} \pm 5^{\circ}($ see $\S 3)$.

Figure 3 shows X-ray light curves of four sources: 4U 1543$47, i=20^{\circ} .7 \pm 1.0 ;$ A $0620-00, i=40^{\circ} .8 \pm 3^{\circ}$; GRO J1655$40, i=70^{\circ} .2 \pm 1{ }^{\circ} 9$; and XTE J1550-564, $i=72^{\circ} \pm 5^{\circ}$. Notice the rather dramatic difference between the two low-inclination systems and the two high-inclination systems. The former sources have "classic" exponential light curves, whereas the latter sources have erratic and more variable light curves. Notice in particular the deep minimum halfway through the light curves of GRO J1655-40 and XTE J1550-564, which have no analog in the low-inclination systems.

The systems shown in Figure 3 are just representative examples, and the reader is invited to look up the X-ray light curves of the remaining seven systems listed in Table 1. Tanaka \& Lewin (1995) and Chen et al. (1997) present pre-1996 light curves, and McClintock \& Remillard (2005) present RXTEera light curves. An inspection of these light curves shows that the six systems with low or moderate inclination (i.e., $i<$ $65^{\circ}$ : 4U 1543-47, A0620-00, GRO J0422+32, GS/GRS 1124683 , GS 2023+338, and GS 2000+25) display simple, classic light curves that rise rapidly and decay exponentially. Admittedly, GS 2023+338 exhibited extreme variability and peculiar behavior; nevertheless, the upper envelope of its light curve resembles a classic light curve (see Fig. 3.2 in Tanaka \& Lewin 1995).

We note that the low-inclination system 4 U $1543-47$ has had three prior outbursts, and there is no evidence for erratic behavior in any of the earlier light curves. Following the 1971 outburst, the smooth exponential decay phase was observed quite continuously for a period of $\approx 120$ days ( $\mathrm{Li}$ et al. 1976). The fast, smooth rise of the 1983 outburst and the early decay period were closely observed for about 8 days with the All-Sky 
Monitor on board Ginga (Kitamoto et al. 1984). No relevant observations of the 1992 outburst have been reported (see Chen et al. 1997). Thus observations of two earlier outbursts of $4 \mathrm{U} 1543-47$ confirm the classic outburst profile shown in Figure 3.

In contrast to the above six sources, the other five systems that are viewed at higher inclination angles near $70^{\circ}$, namely, GRS 1009-45, XTE J1118+480, GRO J1655-40, XTE J1550-564, and SAX J1819.3-2525, all have more complex light curves that display pronounced variability, large dips, and other unusual behavior. Especially telling is the unprecedented light curve of the highest inclination system, SAX J1819.3-2525, with $i=75^{\circ} \pm$ $2^{\circ}$. This source was active at a very low level of intensity $(\sim 0.05$ Crab) for almost a year before it rose abruptly to an intensity of 12.2 Crab. Within $2 \mathrm{hr}$ thereafter, the source returned to its preoutburst intensity (Wijnands \& van der Klis 2000).

The optical light curves of BHXNs also follow a similar progression from simple to complex as the inclination rises above about $70^{\circ}$. We illustrate this fact by briefly comparing the well-sampled optical/X-ray light curves of A0620-00 and XTE J1550-564. A very simple and satisfactory model of the $U B V$ optical light curves of A0620-00 $\left(i=40^{\circ} .8 \pm 3^{\circ}\right)$, which is based on X-ray reprocessing in the accretion disk, is given by Esin et al. (2000). In contrast, the optical light curves of XTE J1550-564 are inscrutable (Jain et al. 2001). In this highinclination system $\left(i=72^{\circ} \pm 5^{\circ}\right)$ the authors find no correspondence between the X-ray thermal accretion disk component and the optical flux. However, the X-ray power-law flux is anticorrelated with optical dip features, which motivates the authors to hazard the exotic suggestion that the optical flux may be upscattered into the X-ray power-law component by the hot corona.

What might cause the correlation of light curve complexity with increasing inclination in BHXNs? Obscuration of some sort is a possible answer. If we accept Milgrom's (1978) explanation of a flared disk to understand the absence of highinclination sources in the observed sample $(\S 3)$, then it is not a big leap to think that the angular extent of the obscuration may vary with time during the outburst. For instance, suppose the flare angle of the disk is $\sim 15^{\circ}$ during the early and late parts of the outburst but increases to $\sim 20^{\circ}$ in the middle of the outburst. Then, for systems with $i$ in the range $\sim 70^{\circ}-75^{\circ}$ we would have a dip in the middle of the light curve whereas there would be little effect for lower inclination systems. In addition, if the flaring angle has short term fluctuations, it could lead to a noisy light curve for high-inclination systems, versus a more smooth light curve for low-inclination systems.

In addition, the radiating gas may be at different heights above the disk at different times during the outburst. For instance, any emission from an extended jet is less likely to be obscured by a flared disk compared to emission from the surface of a thin disk. This looks like a particularly attractive explanation for the strange light curve of SAX J1819.3-2525. We suggest that this system, with the highest inclination angle of all our sources, was strongly obscured most of the time but became bright during a large jet ejection episode when the X-ray-emitting gas was at a relatively large height above the disk. During the rest of the outburst, we probably observed only scattered radiation from the outermost parts of the disk corona, which might explain the very low flux level. Such scattering by a disk corona is observed for several eclipsing LMXBs that contain neutron star primaries (e.g., see White \& Holt 1982; Parmar et al. 1986).

Of course, this discussion is based on a small number of sources - six with low inclinations and five with high inclinations - and the correlation may be a statistical fluke. Interestingly, one could use the same data to argue that the complexity of the light curve increases with increasing binary orbital period. Although the evidence is not as good as for the correlation with inclination angle that we have discussed, it is strong enough to take note. A larger sample will help to sort things out.

\section{SUMMARY}

Starting with the available information on the orbital inclinations of black hole X-ray binaries, we have reached several conclusions in this paper:

1. We find that the quiescent X-ray luminosities of BHXNs show no trend with inclination $i$ (Table 1, Fig. 1). Comparing the observations with the expected trend if the X-ray emission is from a jet oriented perpendicular to the binary orbital plane, and considering in particular the low-inclination source 4U 154347 , we conclude that the bulk Lorentz factor $\gamma$ of the jet is $\leq 1.24$ at the $90 \%$ confidence level and $\leq 1.41$ at the $95 \%$ confidence level. This result for the quiescent state agrees with a similar conclusion reached by Gallo et al. (2003) for the radio-emitting gas and Maccarone (2003) for the X-ray-emitting gas in the low hard state.

2. The source $4 \mathrm{U} 1543-47$ has only an upper limit on its quiescent $X$-ray luminosity. Because of its low inclination angle $\left(i=20^{\circ} .7 \pm 1^{\circ} .0\right)$, this source is most sensitive to relativistic beaming from a jet. We urge greater efforts to measure the quiescent luminosity of the source. A detection at a level well below the present upper limit could be problematic for any jet model that invokes a reasonable velocity for the radiating gas.

3. $4 \mathrm{U} 1543-47$ showed a strong fluorescent iron line with an equivalent width of $300 \mathrm{eV}$ in outburst in the high soft state. Quantifying an argument by Park et al. (2004), we show that the $\mathrm{X}$-ray continuum must have originated in gas with an outward bulk Lorentz factor $<1.04$ (Fig. 2). If this gas was in a jet, then the jet must have been highly subrelativistic. Alternatively, the X-rays could have come from a slowly expanding corona (Beloborodov 1999; Malzac et al. 2001) or from the disk.

4. Of the 11 sources with estimates of $i$ (Table 1 plus the source XTE J1550-564), we show that there are none with $i>75^{\circ}$ or $\cos i<0.25$. The probability that this would happen by chance is only $4 \%$, suggesting that there is a selection effect that prevents edge-on systems from being seen.

5. None of the 20 binaries in our complete sample is an eclipsing source. The probability that it could happen by chance is only $0.5 \%$ (calculated based on the data given in Table 2). This completely independent argument, which moreover does not require estimates of $i$, confirms that high-inclination systems are hard to detect.

6. In our view, the most plausible explanation for the absence of eclipsing sources is that the accretion disks in black hole X-ray binaries are flared (or corrugated or warped) by about $\pm 15^{\circ}$. The disks thus permanently occult the X-ray emission for observers located within $\pm 15^{\circ}$ of the binary orbital plane. Milgrom (1978) suggested the same explanation for the rarity of eclipses in neutron star X-ray binaries.

7. We show that there is a surprising dichotomy between the X-ray light curves of BHXNs with $i \lesssim 65^{\circ}$ and those with $i \sim 70^{\circ}-75^{\circ}$ (Fig. 3). The former sources have smooth wellbehaved outburst light curves with exponential decays, whereas the latter have noisy light curves, often with a deep minimum around the midpoint of the outburst. There are similar indications also in the optical light curves. 
8. We tentatively suggest that the systems with $i \sim 70^{\circ}-75^{\circ}$ have noisy light curves because they are close to being occulted by the disk. Fluctuations in the flaring angle of the disk and/or the height of the X-ray-emitting gas above the disk could cause the observed variations in the flux.

9. The source SAX J1819.3-2525 has the largest inclination angle among all our sources: $i=75^{\circ} \pm 2^{\circ}$. It also has the most extreme X-ray light curve. In this $\mathrm{BHXN}$, we suggest that the primary X-ray source was occulted almost throughout its outburst, with only a small amount of radiation being observed from the outer regions of a large corona. However, for a period of just $2 \mathrm{hr}$, the source became very bright in X-rays. We suggest that during this brief episode there was a powerful jet ejection; $\mathrm{X}$-rays from the jet at a relatively large distance from the black hole were observed directly without being obscured.

We thank Jerry Orosz for advice on using the Eclipsing Light Curve code; we acknowledge the use of the processed $S A S-3$ data from Kenneth Plaks, Jonathan Woo, and George Clark; and we thank Ron Remillard for help with the ASM light curves in Figure 3. This work was supported in part by NASA grants NAG5-10780 and NAG5-9930 and by NSF grant AST 0307433.

\section{REFERENCES}

Abramowicz, M. A., Chen, X., Kato, S., Lasota, J.-P., \& Regev, O. 1995, ApJ, 438, L37

Barret, D., McClintock, J. E., \& Grindlay, J. E. 1996, ApJ, 473, 963

Beloborodov, A. M. 1999, ApJ, 510, L123

Bolton, C. T. 1975, ApJ, 200, 269

Buff, J., et al. 1977, ApJ, 212, 768

Casares, J., Zurita, C., Shahbaz, T., Charles, P. A., \& Fender, R. P. 2004, ApJ, 613, L133

Chaty, S., Mirabel, I. F., Marti, J., \& Rodriquez, L. F. 2001, Ap\&SSS, 276, 153

Chen, W., Shrader, C. R., \& Livio, M. 1997, ApJ, 491, 312

Corbel, S., Nowak, M. A., Fender, R. P., Tzioumis, A. K., \& Markoff, S. 2003, A\&A, 400, 1007

Esin, A. A., Kuulkers, E., McClintock, J. E., \& Narayan, R. 2000, ApJ, 532, 1069

Esin, A. A., McClintock, J. E., Drake, J. J., Garcia, M. R., Haswell, C. A., Hynes, R. I., \& Muno, M. P. 2001, ApJ, 555, 483

Esin, A. A., McClintock, J. E., \& Narayan, R. 1997, ApJ, 489, 865

Esin, A. A., Narayan, R., Cui, W., Grove, J. E., \& Zhang, S.-N. 1998, ApJ, 505, 854

Falcke, H., Körding, E., \& Markoff, S. 2004, A\&A, 414, 895

Fender, R. P. 2005, in Compact Stellar X-Ray Sources, ed. W. H. G. Lewin \& M. van der Klis (Cambridge: Cambridge Univ. Press), in press (astro-ph/ 0303339)

Froning, C. S., \& Robinson, E. L. 2001, AJ, 121, 2212

Gallo, E., Fender, R. P., \& Pooley, G. G. 2003, MNRAS, 344, 60

Garcia, M. R., McClintock, J. E., Narayan, R., Callanan, P., Barret, D., \& Murray, S. S. 2001, ApJ, 553, L47

Gelino, D. M., \& Harrison, T. E. 2003, ApJ, 599, 1254

Gelino, D. M., Harrison, T. E., \& McNamara, J. A. 2001a, AJ, 122, 971

Gelino, D. M., Harrison, T. E., \& Orosz, J. A. 2001b, AJ, 122, 2668

George, I. M., \& Fabian, A. C. 1991, MNRAS, 249, 352

Gies, D. R., Bolton, C. T. 1986, ApJ, 304, 371

Greene, J., Bailyn, C. D., \& Orosz, J. A. 2001, ApJ, 554, 1290

Hameury, J.-M., Barret, D., Lasota, J.-P., McClintock, J. E., Menou, K., Motch, C.,

Olive, J.-F., \& Webb, N. 2003, A\&A, 399, 631

Heinz, S. 2004, MNRAS, 355, 835

Heinz, S., \& Merloni, A. 2004, MNRAS, 355, L1

Heinz, S., \& Sunyaev, R. 2003, MNRAS, 343, L59

Hjellming, R. M., \& Rupen, M. P. 1995, Nature, 375, 464

Hjellming, R. M., et al. 2000, ApJ, 544, 977

Hynes, R. I., Steeghs, D., Casares, J., Charles, P. A., \& O’Brien, K. 2003, ApJ, 583, L95

Ichimaru, S. 1977, ApJ, 214, 840

Jain, R. K., Bailyn, C. D., Orosz, J. A., McClintock, J. E., Sobczak, G. J., \& Remillard, R. A. 2001, ApJ, 546, 1086

Jonker, P. G., \& Nelemans, G. 2004, MNRAS, 354, 355

Kitamoto, S. K., Miyamoto, S., Tsunemi, H., Makishima, \& Nakagawa, M. 1984, PASJ, 36, 799

Kuiper, L., van Paradijs, J., \& van der Klis, M. 1988, A\&A, 203, 79
Li, F. K., Sprott, G. F., \& Clark, G. W. 1976, ApJ, 203, 187

Maccarone, T. J. 2002, MNRAS, 336, 1371

. 2003, A\&A, 409, 697

Malzac, J., Beloborodov, A. M., \& Poutanen, J. 2001, MNRAS, 326, 417

Malzac, J., Merloni, A., \& Fabian, A. C. 2004, MNRAS, 351, 253

Markoff, S., Falcke, H., \& Fender, R. 2001, A\&A, 372, L25

McClintock, J. E., Narayan, R., Garcia, M. R., Orosz, J. A., Remillard, R. A., \& Murray, S. S. 2003, ApJ, 593, 435

McClintock, J. E., \& Remillard, R. A. 2005, in Compact Stellar X-ray Sources, ed. W. H. G. Lewin \& M. van der Klis (Cambridge: Cambridge Univ. Press), in press (astro-ph/0306213 v4)

Merloni, A., Heinz, S., \& di Matteo, T. 2003, MNRAS, 345, 1057

Milgrom, M. 1978, A\&A, 67, L25

Mirabel, I. F., \& Rodríguez, L. F. 1999, ARA\&A, 37, 409

Narayan, R., Barret, D., \& McClintock, J. E. 1997, ApJ, 482, 448

Narayan, R., Mahadevan, R., \& Quataert, E. 1998, in The Theory of Black Hole Accretion Disks, ed. M. A. Abramowicz, G. Bjornsson, \& J. E. Pringle (Cambridge: Cambridge Univ. Press), 148

Narayan, R., McClintock, J. E., \& Yi, I. 1996, ApJ, 457, 821

Narayan, R., \& Yi, I. 1995, ApJ, 452, 710

Orosz, J. A. 2002, in IAU Symp. 212, A Massive Star Odyssey: From Main Sequence to Supernova, ed. K. A. van der Hucht, A. Herrero, \& C. Esteban (San Francisco: ASP), 365

Orosz, J. A., \& Bailyn, C. D. 1997, ApJ, 477, 876

Orosz, J. A., Bailyn, C. D., McClintock, J. E., \& Remillard, R. A. 1996, ApJ, 468,380

Orosz, J. A., \& Hauschildt, P. H. 2000, A\&A, 364, 265

Orosz, J. A., McClintock, J. E., Remillard, R. A., \& Corbel, S. 2004, ApJ, 616, 376

Orosz, J. A., et al. 2001, ApJ, 555, 489 2002, ApJ, 568, 845

Park, S. Q., et al. 2004, ApJ, 610, 378

Parmar, A., White, N. E., Giommi, P., \& Gottwald, M. 1986, ApJ, 308, 199

Pringle, J. E. 1976, MNRAS, 177, 65

Shahbaz, T., Bandyopadhyay, R. M., \& Charles, P. A. 1999, A\&A, 346, 82

Shahbaz, T., Naylor, T., \& Charles, P. A. 1997, MNRAS, 285, 607

Shahbaz, T., Ringwald, F. A., Bunn, J. C., Naylor, T., Charles, P. A., \& Casares, J. 1994, MNRAS, 271, L10

Shapiro, S. L., Lightman, A. P., \& Eardley, D. M. 1976, ApJ, 204, 187

Sutaria, F. K., et al. 2002, A\&A, 391, 993

Tanaka, Y., \& Lewin, W. H. G. 1995, in X-Ray Binaries, ed. W. H. G. Lewin, J. van Paradijs, \& E. P. J. van den Heuvel (Cambridge Univ. Press: Cambridge), 126

Tomsick, J. A., et al. 2003, ApJ, 597, L133

White, N. E., \& Holt, S. S. 1982, ApJ, 257, 318

Wijnands, R., \& van der Klis, M. 2000, ApJ, 528, L93

Yuan, F., Cui, W., \& Narayan, R. 2004, ApJ, submitted (astro-ph/0407612)

Zdziarski, A. A., Lubinski, P., Gilfanov, M., \& Revnivtsev, M. 2003, MNRAS, 342,355 